\title{
Multilayer film shields for the protection of PMT from constant magnetic field
}

\author{
V. V. Dmitrenko, ${ }^{1}$ David Besson, ${ }^{1,2,3}$ PhyoWai Nyunt, ${ }^{1}$ S. S. Grabchikov, ${ }^{2}$ V. M. Grachev, ${ }^{1}$ \\ C. C. Muraviev-Smirnov, ${ }^{1}$ S. E. Ulin, ${ }^{1}$ Z. M. Uteshev, ${ }^{1}$ and K. F. Vlasik ${ }^{1}$ \\ ${ }^{1}$ National Research Nuclear University "MEPhI," Moscow, Russian Federation \\ ${ }^{2}$ Scientific and Practical Center and National Academy of Sciences of Belarus for Materials, \\ Minsk, Republic of Belarus \\ ${ }^{3}$ Department of Physics and Astronomy, University of Kansas, Lawrence, Kansas 66045, USA
}

(Received 26 August 2014; accepted 9 December 2014; published online 7 January 2015)

\begin{abstract}
Photomultiplier tubes (PMTs) are widely used in physical experiments as well as in applied devices. PMTs are sensitive to magnetic field, so creation of effective magnetic shields for their protection is very important. In this paper, the results of measurements of shielding effectiveness of multilayer film magnetic shields on PMT-85 are presented. Shields were formed by alternating layers of a material with high magnetic permeability (Ni-Fe) and high electric conductivity $-\mathrm{Cu}$. The maximum number of bilayers reached 45 . It is shown that in weak magnetic fields up to $0.5 \mathrm{mT}$, the output signal amplitude from PMT-85 does not change for all used multilayer shields. In strong magnetic field of 2-4 mT, the output signal amplitude decrease with 10\%-40\% depending from the number of layers in the shield. The Pulse distribution of PMT- 85 in magnetic field $0.2-4 \mathrm{mT}$ slightly changed in the range $1.1 \%-1.3 \%$ for the case when the number of layers do not exceed 10 and practically did not change for a shield with 45 double layers. (C) 2015 AIP Publishing LLC. [http://dx.doi.org/10.1063/1.4904873]
\end{abstract}

\section{INTRODUCTION}

Magnetic shields are used in various fields of science and technology. Particularly, widespread is the use of magnetic shields in nuclear physics experiments, where widely used photomultiplier tubes (PMTs) are often subjected to strong magnetic fields. ${ }^{1}$ PMTs are important elements of experimental detectors, where they are commonly used as photodetectors. Unfortunately, in the presence of sufficiently strong magnetic fields, the trajectories of photo-electrons in the PMT dinode's system are modified, resulting in compromised performance. Despite the partial successes in reducing the effect of magnetic fields on PMTs by optimization of design, this problem is still not solved completely. Therefore, using shields to protect PMTs from magnetic fields is still relevant.

It has been shown both experimentally ${ }^{2-5}$ and theoretically $^{6-11}$ that multilayer magnetic shields are more effective than single homogeneous monolayers. Particularly promising are shields based on multilayer film nano shields. The use of magnetic shields based on multilayer film nanostructures allows for shielding effectiveness approximately 5-7 times higher than single layer shields of equal thickness based on permalloy or clean steel. ${ }^{12}$ In this paper, the effectiveness of such shields, as applied to PMTs, is discussed.

\section{THE EXPERIMENTAL SET-UP}

Quantification of the shielding effectiveness of multilayer film shields (MFS) was carried out by studying the response of the PMT- 85 photomultiplier. To study the magnetic field effect on the performance of the PMT, the PMT was placed into a magnetic field between two permanent magnets. The magnetic field at the location of the photomultiplier was measured using a Hall sensor and varied from 2 to 30 gauss due to the change in the distance between the magnets. A Light-emitting diode (LED) was fixed to the PMT via a bushing, which eliminated light bleeding into the PMT. The light from the LED was subsequently channeled directly to the photocathode. Current pulses were applied to the LED using the G5-56 signal generator. The photomultiplier supply voltage was set to $800 \mathrm{~V}$ using the BHV2-95 HV supply. Using the amplifier-shaper and pulse analyzer (SBS-50M company "Green Star"), ${ }^{13}$ which are pre-loaded with the software analysis package "PMT," the spectrum of electrical pulses from the LED was recorded. Fig. 1 summarizes the experimental setup.

To determine the influence of magnetic field on the PMT85 performance, a series of measurements of the dependence of gain and pulse resolution of the PMT were made, under the following conditions: PMT without a shield, PMT in a simple aluminum jacket (internal diameter $37 \mathrm{~mm}$, length $130 \mathrm{~mm}$, thickness of wall $1 \mathrm{~mm}$ ), PMT with a permalloy shield $0.5 \mathrm{~mm}$ consisting of five layers of foil with a $0.1 \mathrm{~mm}$ thickness, coating of the aluminum jacket, PMT with three multilayer film shields with different numbers of layers which coated the aluminum jacket. MFS were formed by electro deposition method and uniformly covered the outer surface of the aluminum jacket along all its length (see Table I).

\section{RESULTS OF PMT TEST IN A MAGNETIC FIELD WITHOUT SHIELD}

Initially, measurements were made of the influence of the magnetic field on the amplitude of the output signal from an unshielded PMT-85 when exposed to a magnetic field, along the three mutually perpendicular axes of the PMT. Orientation of the PMT relative to axes shown in Fig. 2. 


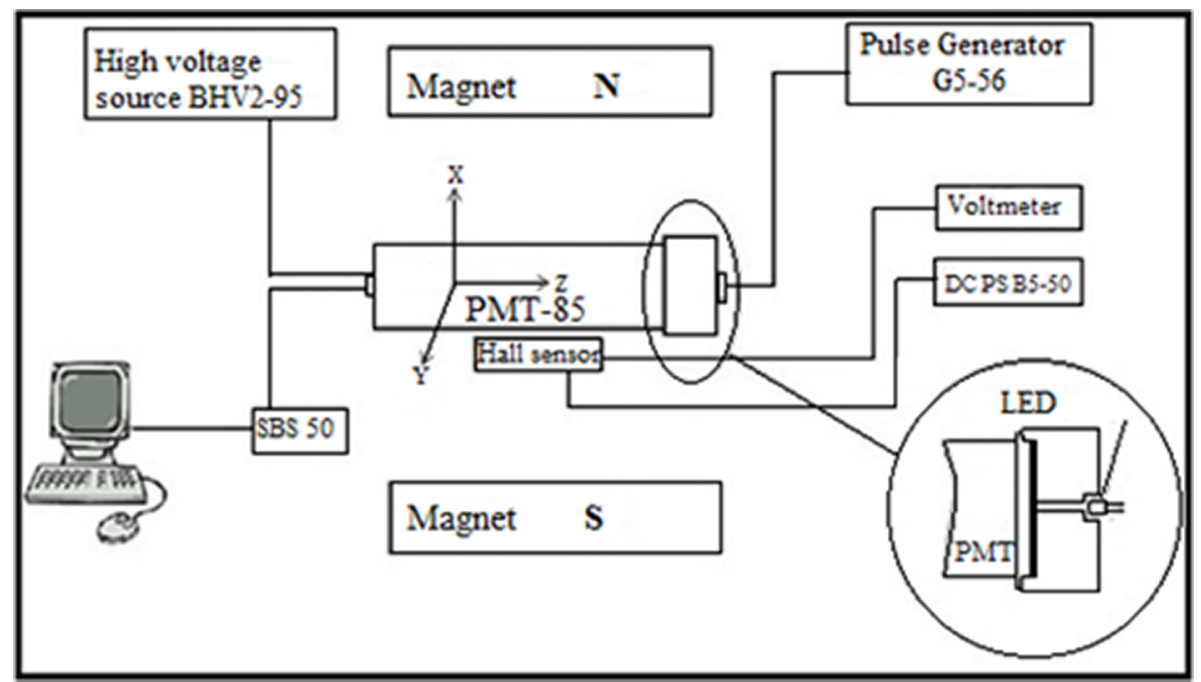

FIG. 1. Experimental setup.

We measured the relative amplitude of the output signal $\left(\mathrm{U} / \mathrm{U}_{0}\right)$ and the pulse amplitude resolution output from the PMT $\left(\mathrm{U}_{0}\right.$ - output signal amplitude of the PMT without magnetic field, U—output signal amplitude of the PMT with applied magnetic field). Our measured values $\left(\mathrm{U} / \mathrm{U}_{0}\right)$ for unshielded PMT-85 perpendicular and parallel to the external magnetic field are shown in Fig. 3.

In weak magnetic fields with induction of $0.1-0.5 \mathrm{mT}$ for cross and parallel orientations, we observe a reduction in the amplitude of output signal by $20 \%$ and $1 \%$ from the maximum, respectively. In magnetic fields of 2-3 $\mathrm{mT}$, the amplitude of the output signal decreases by $95 \%$ (perpendicular orientation) and $60 \%$ (axial orientation).

The results of these measurements confirm the strong dependence of the gain of the PMT-85, even at relatively low magnetic field strengths, especially when it oriented perpendicular to the $\mathrm{Z}$ axis. This again emphasizes the need to use magnetic shields to ensure efficiency of the PMT in the presence of a magnetic field.

\section{RESULTS ON THE PMT-85 MAGNETIC FIELD SHIELDING EFFECTIVENESS USING MULTILAYER NANOSHIELDS}

The shielding effectiveness of the above shields was held for two orientations of the magnetic field relative to the shield: perpendicular to the $\mathrm{Z}$ axis and along the $\mathrm{Z}$ axis of the PMT.

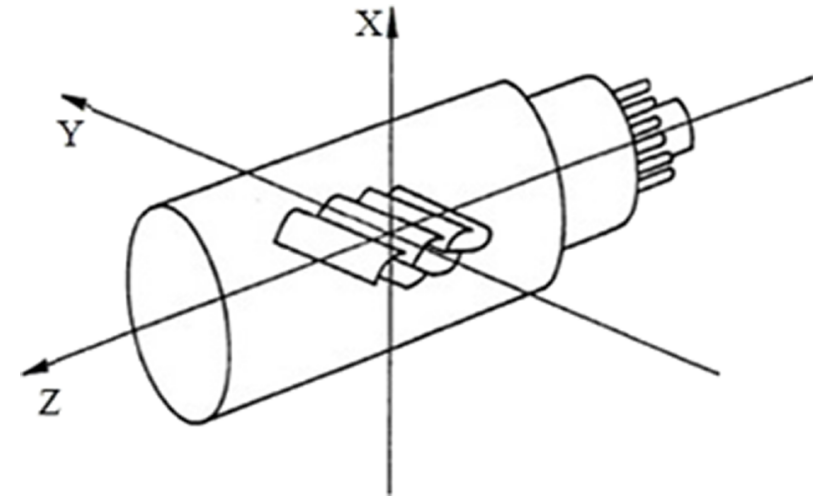

FIG. 2. Coordinate system of the photomultiplier tube.

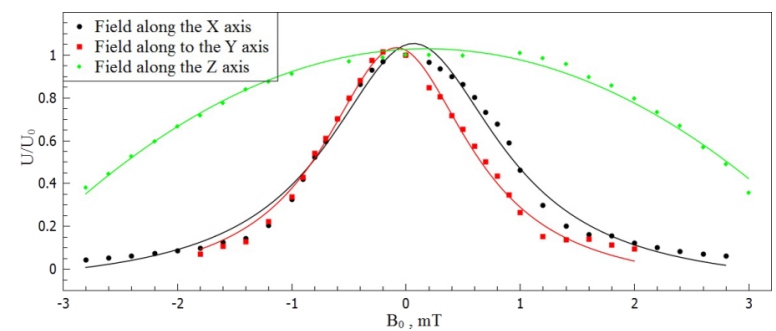

FIG. 3. Dependence of the relative amplitude of the output signal of an unshielded PMT-85 on the magnetic field along the axes $(\mathrm{X}, \mathrm{Y})$ and parallel to the axis $(\mathrm{Z})$.

TABLE I. Characteristics of sample shields.

\begin{tabular}{|c|c|c|c|c|c|c|c|}
\hline $\begin{array}{l}\text { Sample } \\
\text { Shield }\end{array}$ & $\begin{array}{l}\text { Soft magnetic } \\
\text { layer thickness } \\
\qquad(\mu \mathrm{m})\end{array}$ & $\begin{array}{c}\text { Total number of } \\
\text { soft magnetic } \\
\text { layers }\end{array}$ & $\begin{array}{c}\text { Total thickness } \\
\text { of soft magnetic } \\
\text { layers }(\mu \mathrm{m})\end{array}$ & $\begin{array}{c}\text { Copper layer } \\
\text { thickness }(\mu \mathrm{m})\end{array}$ & $\begin{array}{l}\text { Total number of } \\
\text { copper layers }\end{array}$ & $\begin{array}{c}\text { Total } \\
\text { thickness of } \\
\text { copper layers } \\
(\mu \mathrm{m})\end{array}$ & $\begin{array}{c}\text { Total } \\
\text { shield } \\
\text { thickness } \\
(\mu \mathrm{m})\end{array}$ \\
\hline MFS no. 1 & 150 & 3 & 450 & 5 & 2 & 10 & 460 \\
\hline MFS no. 2 & 45 & 10 & 450 & 5 & 9 & 45 & 495 \\
\hline MFS no. 3 & 10 & 45 & 450 & 5 & 44 & 220 & 670 \\
\hline Permalloy & 100 & 5 & 500 & N/A & N/A & N/A & 500 \\
\hline
\end{tabular}




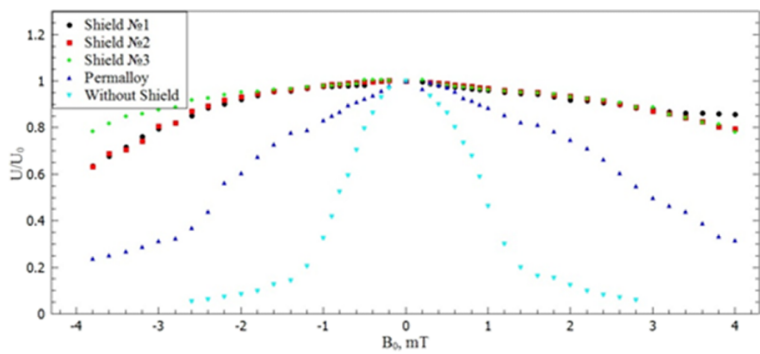

FIG. 4. The relative amplitude of the PMT-85 output signal, for different types of shields, depending on the external magnetic field in the direction of the magnetic field perpendicular to the $\mathrm{Z}$ axis.

The intensity of applied magnetic field was varied from 0 to $4 \mathrm{mT}$.

Fig. 4 displays the dependence of the relative output signal amplitudes of the PMT-85 on the magnetic field, perpendicular to the $\mathrm{Z}$ axis with various types of shielding. In practice, all studied samples of MFS eliminate the negative effect of a magnetic field values up to $0.5 \mathrm{mT}$, with a decrease in $\mathrm{U}$ no more than $1 \%-2 \%$. For magnetic fields in the range of values 2-4 $\mathrm{mT}$, and depending on the MFS type, the decrease in U may reach $10 \%-40 \%$. The best shielding was obtained with sample no. 3, for which the decrease in PMT output signal amplitude, at $2 \mathrm{mT}$, did not exceed $10 \%$.

The shield made from permalloy does not provide sufficient protection for the PMT. The measured reduction in $\mathrm{U}$ for a magnetic field of $0.1-0.5 \mathrm{mT}$ focused perpendicular to the $\mathrm{Z}$ axis reached $5 \%-10 \%$; however, for a magnetic field of $2-3 \mathrm{mT}$, this value rose sharply to $50 \%-80 \%$.

Evaluation of the effect of shielding on the output characteristics of the PMT-85 was also carried out by measuring the amplitude resolution of the pulses from the photomultiplier. Fig. 5 shows the dependence of the amplitude resolution of pulses distribution $(\mathrm{K})$ on magnetic field for PMT-85 with different magnetic shields.

$$
K=\frac{\Delta U}{U_{\max }},
$$

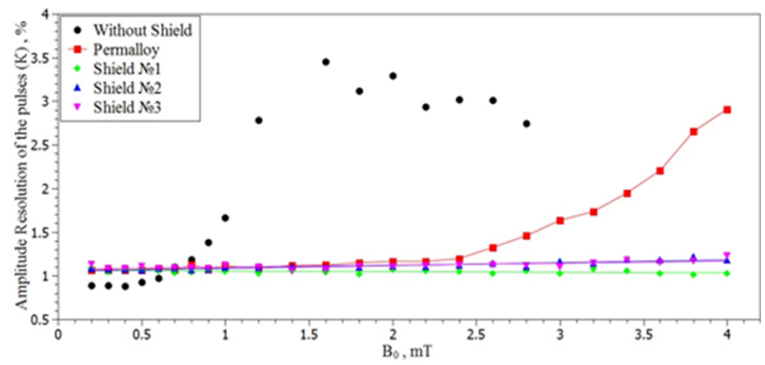

FIG. 5. Dependence of the amplitude resolution of PMT-85 pulses on the induction of magnetic field without a shield and also with shields of permalloy and MFS nos. 1-3, with direction of the magnetic field perpendicular to the $\mathrm{Z}$ axis. where $\mathrm{K}$ is the amplitude resolution of pulses distribution, $\Delta U$ is the full width at the half-height distribution, $U_{\max }$ is the amplitude at the peak of distribution.

The data show that for unshielded of PMT-85, the K value is degraded by $20 \%$ in magnetic fields at an induction up to $1 \mathrm{mT}$ and decreases by a factor of $2.5-3$ as the induction increases to $1-2.5 \mathrm{mT}$.

The amplitude resolution of PMT-85 pulses with a permalloy shield was virtually unchanged for magnetic fields up to magnitude $2.5 \mathrm{mT}$. However, increasing the magnetic field from 2.5 to $4 \mathrm{mT}$ leads to degradation in performance by a factor of 2.5 . The most stable results for the amplitude resolution of PMT-85 pulses are obtained using MFS shields. In magnetic fields with induction of $0.2-4 \mathrm{mT}$, the $\mathrm{K}$ values for MFS sample nos. 2 and 3 only slightly vary from $1.1 \%-1.3 \%$. The amplitude resolution for MFS sample no. 1 is practically unchanged up to $4 \mathrm{mT}$.

\section{CONCLUSION}

Our result demonstrates that shields based on multilayer film structures are more effective shields than those constructed from permalloys and allow the use of PMTs in magnetic fields up to $2 \mathrm{mT}$, which makes it possible to apply them successfully in scientific equipment on-board spacecraft and also ground-based experiments, particularly in modern particle accelerators.

\section{ACKNOWLEDGMENTS}

The author would like to thank Professor A. M. Galper for his interest in this investigation and his helpful comments.

${ }^{1}$ E. Desoppere and G. Van Oost, Rev. Phys. Appl. 18, 359-361 (1983).

${ }^{2}$ W. Esmarch, Ann. Phys. 39, 1540-1552 (1912).

${ }^{3}$ W. Esmarch, Ann. Phys. 39, 1553-1566 (1912).

${ }^{4}$ S. M. Frake and T. L. Thorp, "Shielding of low magnetic fields with multiple cylindrical shells," Rev. Sci. Instrum. 42(10), 1411 (1971).

${ }^{5}$ I. Sasada, T. Yamamoto, and T. Yamauchi, "Large shielding factor obtained by a multiple-shell magnetic shield having separate magnetic shaking," J App. Phys. 79, 5490 (1996).

${ }^{6}$ W. G. Wadey, "Magnetic shielding with multiple cylindrical shells," Rev. Sci. Instrum. 27(11), 910 (1956).

${ }^{7}$ A. J. Mager, IEEE Trans. Magn. 6, 67-75 (1970).

${ }^{8}$ D. U. Gubser, S. A. Wolf, and J. E. Cox, Rev. Sci. Instrum. 50, 751-756 (1979).

${ }^{9}$ D. Dubbers, Nucl. Instrum. Methods Phys. Res., Sect. A 243, 511-517 (1986).

${ }^{10}$ T. J. Sumnert, J. M. Pendleburys, and K. F. Smith, "Conventional magnetic shielding,” J. Phys. D: Appl. Phys. 20, 1095-1101 (1987).

${ }^{11}$ E. Paperno, M. V. Romalis, and Y. Noan, "Optimization of five-shell axial magnetic shields having openings in the end-caps," IEEE Trans. Magn. 40(4), 2170 (2004).

${ }^{12}$ S. S. Grabchikov and E. V. Kapralova, "Screens for static magnetic fields on the basis of electrolytically deposited multilayer films," Report No. AC34 (Scientific and Practical Center and National Academy of Belarus for materials science, 2012).

${ }^{13}$ See www.greenstar.ru for the group of companies "Green Star." 